\title{
Patients' Perceptions of Having a Good Life One Year after Arthritis Patient Education: A Qualitative Study Nested within a Randomized Controlled Trial
}

\author{
Kjersti Grønning ${ }^{1}$, Ola Bratås², Aslak Steinsbekk ${ }^{3}$ \\ ${ }^{1,2,3}$ Department of Public Health and Nursing, Faculty of Medicine and Health Sciences, Norwegian \\ University of Science and Technology (NTNU), Norway \\ Corresponding Author: Kjersti Grønning (kjersti.gronning @ntnu.no)
}

\begin{abstract}
Background: Patients with inflammatory polyarthritis have various degrees of diseaserelated challenges such as joint pain, stiffness, fatigue, and physical limitations. Despite these challenges, patients strive for a good life using their personal resources, often taught in patient education. The effect of patient education in polyarthritis is well studied; however, long-term studies on what patients perceive as a good life after participating in arthritis patient education are scarce.

Purpose: The aim of this study was to explore patients' perceptions of having a good life one year after attending a nurse-led patient education intervention.

Methods: This was a qualitative study nested within a randomized controlled trial (RCT) studying the effect of nurse-led patient education. Fifteen individual semistructured interviews were conducted among those who attended the nurse-led patient education intervention. The intervention focused on the consequences of living with chronic inflammatory arthritis. The interviews explored how and whether the intervention had made any changes in the informants perceived health, well-being, arthritis, flares, and treatment regimes. The data were analyzed thematically using systematic text condensation.

Results: The findings showed that the informants' perceptions of having a good life were related to a stable disease with few symptoms, effective treatment regimes, discovering new opportunities and perspectives in life, as well as making choices that felt right. Creating a good life was something the informants had acquired knowledge about in the nurse-led patient education intervention, particularly in the part where they had discussed and shared experiences with each other on how they managed their arthritis in different ways and settings.

Conclusion: Participating in a nurse-led patient education intervention in arthritis helped the informants to discover new opportunities and perspectives on creating a good life, especially through exchanging experiences with fellow patients.
\end{abstract}

Keywords: Chronic arthritis; well-being; health promotion; patient education; qualitative methodology 


\section{BACKGROUND}

People living with inflammatory polyarthritis such as rheumatoid arthritis (RA) or psoriatic arthritis (PsA) experience shifting and fluctuating symptoms of pain, joint stiffness and fatigue. The diseases have an impact on patients' well-being (Poh, Chan, Lee, Lahiri, Mak \& Cheung, 2016) and lead to numerous challenges related to physical functioning and maintenance of social roles (Daker-White, Donovan, \& Campbell, 2014). Despite challenges, patients strive for a good life by using their personal resources and support from others (Bergsten, Bergman, Fridlund, \& Arvidsson, 2011).

A key function for nurses (Henderson, 2006) is to make patients able to handle their disease on their own. If patients are able to gain control over their health, they can make choices that are conducive to their health and live good lives (Sharpe, 2016). To do so, nurses can focus on patients' health resources and self-management skills in patient education (PE) (Cottrell et al., 2012; van Eijk-Hustings et al., 2011). Patient education (PE) in inflammatory polyarthritis is valuable in supporting patients to cope with their disease (Albano, Giraudet-Le Quintrec, Crozet, \& d'Ivernois, 2010; Cottrell et al., 2012; Dures, et al., 2016; Poh et al., 2016; Stenberg, Haaland-Overby, Fredriksen, Westermann, \& Kvisvik, 2016). A recent review pointed out that reduced symptom burden, greater awareness, improved self-management strategies, peer support, learning, and hope were beneficial outcomes from PE (Stenberg et al., 2016). The aim of PE, in general, is not only for knowledge transfer and disease control but also enabling patients to handle their disease, adjust to the condition and maintain a good quality of life (de Ridder, Geenen, Kuijer, \& van Middendorp, 2008).

Nurses focusing on patients' health resources and self- management skills (Cottrell et al., 2012; van Eijk-Hustings et al., 2011) are in the scope of health promotion (WHO, 1986), which is also prominent in the European League Against Rheumatism's (EULAR) recommendations for PE in people with inflammatory arthritis (Zangi et al., 2015). These recommendations state that the overall aim of PE is to enable patients to manage their lives with arthritis. PE has a potential to support patients to optimize their health and well-being and not being limited by their disease. The effects of PE in patients with inflammatory arthritis are well studied (Albano et al., 2010; Stenberg et al., 2016), but knowledge about patients' perceptions of having a good life, and how PE influences these perceptions are scarce (Albano et al., 2010). In order to improve PE, patients' viewpoints are essential (Zuidema, Repping-Wuts, Evers, Van Gaal, \& Van Achterberg, 2015).

\section{PURPOSE}

The aim of this study was to explore patients' perceptions of having a good life one year after attending a nurse-led PE intervention for patients with chronic inflammatory polyarthritis. The nurse-led PE intervention was found effective on patients' well-being after 12 months (Grønning, Rannestad, Skomsvoll, Rygg, \& Steinsbekk, 2013).

\section{METHODS}

This was a qualitative study nested within a randomized controlled trial (RCT) (Grønning et al., 2013) aiming to explore patients' descriptions of what constituted a 
good life after attendance in nurse-led PE, and if PE had any influence on patients' descriptions. The PE intervention consisted of a combination of three group sessions with 8-10 patients followed by an individual educational session approximately 1-2 weeks after the group sessions. The content in the PE included lectures and discussions about the arthritis process, symptoms, self-management, problem solving, how to live with arthritis, emotions, motivation, goal setting, medical treatments, side effects, healthy lifestyles, exercise, food/diet and resources (Grønning, Skomsvoll, Rannestad, \& Steinsbekk, 2012). The study was conducted in accordance with the Declaration of Helsinki, and the Regional Ethics Committee in Medicine, Central Norway approved the study (4.2007.2472).

\section{Informants}

The informants $(n=15)$ were recruited among the participants included in the RCT (Grønning et al., 2013). The inclusion criteria for the qualitative study were the same as for the RCT. The informants had a chronic inflammatory joint disease (RA, PsA or unspecified polyarthritis (UA)), were between 18 to 80 years old, and were able to participate in the nurse-led PE intervention. The informants had been interviewed previously; before they attended the nurse-led PE intervention (Grønning, Lomundal, Koksvik, \& Steinsbekk, 2011) and 8-10 weeks after the intervention was finished (Grønning, Midttun, \& Steinsbekk, 2016).

\section{Data collection}

The first author (a trained interviewer) performed the individual face-to-face interviews in the period from January to March 2010. The interviews were tape-recorded and took place right after the 12-month follow-up data collection for the RCT (Grønning et al., 2013). Before the interviews were conducted, the research team developed a thematic interview guide (Table 1) based on a literature discussion and previous interviews. The main questions were open-ended. Depending on how freely the informants spoke, the interviewer followed up by probing sub-questions (e.g. could you tell more about that, could you give an example, is there anything else you would like to add) to get more and richer data.

The interview results at baseline (Grønning et al., 2011) and eight months earlier (Grønning et al., 2016) were read before each informant was interviewed, to state what the informants had said about how they experienced to live with arthritis at different time points. The rationale behind this was to help the informants concentrate their experiences to what had happened in their lives between the time points, how they managed their arthritis now, and if the nurse-led PE intervention had influenced their experiences in anyways.

Table 1. Interview guide at 12 months

\begin{tabular}{ll}
\hline Theme & \multicolumn{1}{c}{ Questions } \\
\hline Changes & Have you experienced any changes in the situation since last time \\
& we spoke? (Disease flares, treatment regimes, new drugs, other \\
& non-pharmacological interventions.) \\
& - Have the changes influenced your well-being in anyways?
\end{tabular}


Nurse Media Journal of Nursing, 7(1), 2017, 4

\begin{tabular}{|c|c|}
\hline Theme & Questions \\
\hline & Describe how. \\
\hline A good life & $\begin{array}{l}\text { - What makes you feel good? } \\
\text { - What do you do to feel good? }\end{array}$ \\
\hline $\begin{array}{l}\text { Learning } \\
\text { experiences }\end{array}$ & $\begin{array}{l}\text { - What did you learn from participating in the PE program? } \\
\text { - Has the PE program made any differences in your life, please } \\
\text { describe how? }\end{array}$ \\
\hline
\end{tabular}

\section{Data analysis}

The data were analyzed using a systematic text condensation (STC) (Malterud, 2012). STC is a descriptive thematic cross-case analysis strategy that is suited for presenting experiences as expressed by the subjects being interviewed (Malterud, 2012). The analyses consisted of four steps, starting to read all the transcripts to get an overall impression of the interviews and identifying preliminary themes associated with the informants' perceptions of having a good life. Then, the first author identified and coded the meaning units, sorted the meaning units into categories, and related the categories to the preliminary themes. The authors discussed and refined the categories through several meetings, followed by process of condensation and summarized the data into generalized descriptions of what constituted a good life one year after attending the nurse-led PE intervention.

To ensure a correct representation of the informants' voices, the first author went back to the initial transcripts. Parallel to the analyses process, the research team searched for and went through the new literature on health promotion, self-management, and patient education. The analysis was data-driven within a health promotion framework as a template (WHO, 1986), focusing on health as a positive concept emphasizing social and personal resources as well as physical capacities. On this basis, the analysis was concentrating on what enables people to improve their health, how to identify and realize desires, satisfy needs, and cope with one's situation. To systematize the transcripted data, the data management program of NVivo 11.0 (QSR, 2007) was used.

\section{RESULTS}

The sample consisted of 4 males and 11 female patients from 38 to 77 years old. The informants had different length of education, employment status, diagnosis, disease duration and medical treatment regimes. Table 2 presents the characteristics of the informants.

Table 2. Informant characteristics $(n=15)$

\begin{tabular}{lc}
\hline Characteristics & $\mathrm{N}(\%)$ or mean (range) \\
\hline Female & $11(73)$ \\
Male & $4(27)$ \\
Age & $58(38-77)$ \\
University & $4(27)$ \\
Middle & $5(33)$ \\
Compulsory school & $6(40)$ \\
\hline
\end{tabular}




\begin{tabular}{lc}
\hline Characteristics & $\mathrm{N}(\%)$ or mean (range) \\
\hline Employment (part or full-time) & $2(13)$ \\
RA & $10(67)$ \\
PsA & $4(27)$ \\
UA & $1(6)$ \\
Diseases duration in years & $10(2-20)$ \\
Using DMARD & $11(73)$ \\
\hline RA=Rheumatoid Arthritis, PsA= Psoriatic arthritis, UA= unspecified polyarthritis, DMARD=Disease \\
modifying antirheumatic drugs
\end{tabular}

The informants expressed that participating in the nurse-led PE intervention was useful because it helped them see new opportunities and perspectives on what constituted a good life. Some said they did not exactly remember what they had talked about and learned in the intervention, while others remembered concrete things and gave examples of what they had learned. The informants said they learned about the immune system, how chronic illness could affect the whole family, how to apply for welfare support, and how others managed their disease, places to go to for rehabilitation, normal symptoms in RA (fatigue, pain), different kind of medications, balance between activity and resting, personal limits or capacity, food, diet and technical aids.

The informants' related having a good life to a stable disease with few symptoms. At the time of the interview, many were generally satisfied with their lives even though arthritis influenced many dimensions of their lives. They spoke about their lives with arthritis as a journey that started when diagnosed. Even though the interviews aimed for the informants' experiences related to the last year, they often started to talk about the beginning of their disease-journey to shed light on the changes they had experienced this past year.

Table 3 presents an overview of how the informants described having a good life one year after attending the nurse-led PE intervention.

Table 3. Overview of the findings

\begin{tabular}{ll}
\hline Categories & Sub- categories \\
\hline It is motivating to discover something new & New knowledge \\
& Useful to others \\
& Friendly environment \\
Effective treatment is essential to feel good & Medical treatment regimes \\
& Non-medical treatment options \\
Making choices that feel right & Possibilities \\
& Limitations \\
\hline
\end{tabular}

\section{It is motivating to discover something new}

The informants spoke about acquiring new knowledge from discussions and lessons in the nurse-led PE intervention. Sharing experiences of living with arthritis with other patients were especially helpful. The discussions on how to live with arthritis felt reassuring because the informants experienced that their invisible symptoms of pain and 
tiredness were acknowledged; the symptoms they had were confirmed as real, normal, but not dangerous or life threatening. Listening to other patients' stories added something extra, and it made it easier to accept the reality. Some informants said they felt better because they discovered that they had fewer problems with their arthritis compared to other patients in the group. Others said it was motivating to see that other patients did well. One informant said "Talking to others made me accept the situation, it was normal to feel the way I did" (Male, PsA for 17 years).

Although a few informants said they did not learn anything new about arthritis as a disease, they still experienced the discussions about living with arthritis as meaningful. One informant highlighted that the group discussions had initiated a positive process that made her feel more confident in how to handle her arthritis. Others expressed how knowledge about practical advice was useful, while some said they learned new things about applying for welfare support, different technical aids that could be useful, medical treatment options, the necessity of asking for help, and that tiredness was a common and normal symptom.

Informants who had lived with arthritis for several years said they got positive feedback from the rest of the group when they talked about how they had handled their arthritis throughout the years. The feedback made them discover that they were valuable persons and a resource to other patients. These experiences made them feel good. One of the informants expressed "I noticed that my experiences were useful to the others. I got impulses from them. I think it was smart to have a group like this; it was very nice" (Male, RA for 14 years).

The informants also expressed that knowing more about arthritis made them more confident in how to handle their arthritis and they became hungry for more information after the nurse-led PE. The explained how they had searched for more knowledge and that they preferred literature that was scientific but written for ordinary people. One informant, in particular, said he had stopped searching for alternative therapies after the PE because he had learned something important about the immune system. He explained that now he knew that it could be dangerous to combine certain kinds of alternative therapies and disease modifying anti-rheumatic drugs (DMARDs). Before attending the nurse-led PE intervention, the informant used to think that increasing the immune system with herbs was important to beat arthritis.

As a final point, the informants pointed to the nurses as essential in the PE because they facilitated a friendly atmosphere. The atmosphere was essential for feeling comfortable in sharing experiences and asking questions. The nurses asked relevant questions and initiated good discussions, which gave the informants a positive drive to continue working with their personal goals. The informants also said it was reassuring to get information about where to call if they had questions to ask. One informant expressed what he had learned in the nurse-led PE intervention like this "I got a deeper insight in the "mystery" of arthritis" (Male, PsA for 17 years). 


\section{Effective treatment is essential to feel good}

The informants stated that they did not consider themselves as patients or "being sick" as long as the arthritis was stable and controlled. Effective medications were essential to keep the arthritis stable with minimum symptoms. In periods when arthritis gave them few or no symptoms, the only thing that reminded them of having a chronic disease, was when they had to take their medications. On the other hand, in addition to having periods when the arthritis was stable, many of the informants had experienced that when the medications had stopped working, it caused more pain, loss of energy, bad sleep, depraved mood and a feeling of being ill. In these cases, the informants said they always believed that the doctors would come up with new suggestions of effective medications. How to handle changes occurring from ineffective medications and side effects was also a theme the informants learned about and discussed in the PE. They emphasized that knowing more about how to handle such incidents was both useful and comforting. One informant expressed "I was not afraid. I have experienced side effects of my medications earlier, but I got angry because it happened again" (Female, RA for 9 years).

Some informants said they did not want to think about themselves as a person with a chronic disease because they felt more disabled or sick if they thought too much about that. They treated themselves by changing focus to things they were able to do and carrying out activities they enjoyed, e.g. reading books, painting, drawing, playing or listening to music, being physically active, doing voluntary stuff, using dark humor or spending more time with family and friends. After the discussions with the other participants, one informant said she was motivated not to give up when arthritis flared. She said "It (the group discussion) was very good! One patient knows something that the others don't know, and vice versa... When you hear it from the patient directly, it means something extra. It was very smart" (Female, RA for 20 years).

Several informants had regular visits to a physiotherapist in addition to exercising on their own. They had experienced that different physical activities such as water gymnastics, relaxing exercises, walking, skiing, etc. alone or together with others made them feel better, refilled them with energy and improved their mental health. However, some informants declared that their motivation to exercise was not always present. They pushed themselves to carry out exercises because they felt obligated to, knowing that it felt good afterward. One informant expressed "Once you have come to a point where you notice that it helps, and then, it somehow, is a pleasure[..] Now I feel in better physical shape, and it affects my mental health in a positive direction" (Male, PsA for 17 years).

\section{Making choices that feel right}

Many of the informants were not in paid employment, but whether or not it was right to continue working was a topic raised by the informants. The informants spoke about valuable discussions focusing on what was the right choice for them. One informant explained how she had gone through a long and difficult process until she managed to conclude that she needed to prioritize her health rather than struggling to stay in her job. Another informant had come to a different solution. If he prioritized to exercise regularly, he got more energy and was able to work. Additionally, he spoke about a 
having a good cooperation with his employer who made extra efforts to make it possible for him to work. The employer facilitated both flexible working hours and flexibility in when he had to be present. The informants emphasized that the nurses and other participants in the PE group supported both solutions (to stop or to continue work) when discussing employment in the nurse-led PE intervention. One informant in the middle of a process of deciding whether she should continue to work or not said the discussions with the rest of the group made her more comfortable in making her decision. She stopped working but kept in touch with her colleagues since they had become important people in her life. Other informants, who had made similar choices earlier on, said that keeping in touch with former colleagues was important for maintaining a social network. "My colleagues want me back, and maybe I can work a little bit, take some shifts after I have been on disability benefits for a year. So now, I go to my exercise class three days a week and meet my colleagues in café when they have a day off" (Female, PsA for two years).

The informants also talked about how prioritizing and making choices between daily tasks could be difficult, e.g. working, house maintenance tasks, social activities and exercising. Being aware of their personal capacity required hard work and concentration. If the informants pushed themselves too hard, they could end up lying on the coach the next day. However, the informants had realized that taking one-step at a time was necessary. Some said they knew this before attending the nurse-led PE intervention while others explained how the discussions about balancing between being active, resting and allocating new energy had helped them to prioritize better what to do or not. One informant said "I am aware of the fact that I have to pay attention to the disease. I have to set limits for what I manage to do. [....] I concentrate on the things I can do, and I do not think about the stuff I cannot do" (Female, RA for 20 years).

\section{DISCUSSION}

This study adds important and new knowledge about what and how patients with inflammatory polyarthritis make use of knowledge learned one year after participating in nurse-led PE. Through participation in the PE, the informants discovered new opportunities on how creating a good life despite having arthritis. They used the knowledge, experienced that learning new things was motivating, and it helped the patients to make choices that felt right for the individual patient. Findings in this study also confirm already existing knowledge about the value of patients sharing their experiences about managing chronic illness (Ring Jacobsson, Milberg, Hjelm, \& Friedrichsen, 2016). Particularly the dialogs on how others managed their disease and what composed normal symptoms in arthritis were valued in this study.

\section{Having a good life}

This study displayed that knowledge on how to make use of one's resources to adjust to new situations is essential for patients with arthritis to have a good life. This finding is in line with other studies targeting how patients cope and manage their lives with arthritis (Bergsten et al., 2011; de Ridder et al., 2008; Dures et al., 2016; Dures et al., 2012). The analyses also revealed that the informants did remember and used the knowledge they learned one year earlier to manage their disease. The discussions with other participants and nurses acknowledged the informants' symptoms and problems 
related to having arthritis and helped the informants focusing on possibilities instead of limitations. According to the WHO, a perception of having good health is a major resource for social, economic and personal development and an important dimension of quality of life (WHO, 1986).

The literature on self-management states that patients need to know why and how to manage chronic illness (Schulman-Green, Jaser, Park, \& Whittemore, 2016). This study explored what patients need to know, why, and how they can use knowledge to live a good life with arthritis. The analyses revealed how the informants related their actions and behaviors to the information learned in the nurse-led PE intervention to live a good life. According to the literature on health promotion (Lindstrom \& Eriksson, 2005) and underpinned by several studies on PE for patients with inflammatory polyarthritis (Barlow et al., 2009; Zangi et al., 2015), diabetes (Anderson \& Funnell, 2010) and across different chronic diseases (Stenberg et al., 2016), knowing how to make use of one's own resources and capacity is essential to create good health. Supporting patients to live good lives by deliberating how to manage their illness is also in line with the overarching goal of patient education started decades ago (WHO, 1986), and a key task, function, and goal for clinical nurses (Henderson, 2006).

Our findings also demonstrated that the informants valued and benefitted from the discussions on living with arthritis, including the possible impact arthritis might have on patients' identity when changing employment status (Lempp, Scott, \& Kingsley, 2006). Being aware of the significant risk of work disability in patients with inflammatory arthritis, Burton, Morrison, Maclean, \& Ruderman (2005) proposed employment and possible adjustments at work as an important theme that needs to be discussed in PE. Patients need information on how to make adjustments, emotional support to deal with potential changes in employment status, and overall practical support (Zuidema et al., 2015). Several studies underpin that integrating these issues in PE are essential because being able to work has a positive influence on patients' quality of life (Gronning, Rodevand, \& Steinsbekk, 2010; Holland \& Collins, 2016). Furthermore, talking to others about feelings and emotions related to work loss may be important to deal with sorrow, until the person is ready and discovers how to manage a new life and identity without a job (Zuidema et al., 2015). The Ottawa charter for health promotion (WHO, 1986) emphasizes the importance of creating a new life and identity through developing patients' skills and abilities to identify and realize their aspirations. By enhancing patients' skills, it increases their possibilities to take more control over their own health and environments and to make choices conducive to health. The ideas from health promotion are parallel to the objectives of this nurse-led PE intervention (Grønning et al., 2012).

\section{Sharing experiences}

The value of sharing experiences, which included both newly diagnosed and experienced patients, were clearly the most appreciated impact from the nurse-led PE intervention. This finding confirms that the rationale behind the program (Grønning et al., 2012) was accomplished. The program was built upon the idea that the participants should have space and time for sharing experiences, and for discussing and learning from each other on how and what to do to live a good life when having inflammatory 
arthritis. However, the informants also underlined that the nurses' role was substantial by facilitating a nice friendly atmosphere, asking relevant questions and initiating good discussions. The nurses' behaviors along with the comforting climate in the patient group gave the informants a positive drive to continue working with their goals on their own. A recent qualitative study in cardiac PE (Svavarsdottir, Sigurdardottir, \& Steinsbekk, 2015) also found that the personnel in charge was essential in establishing interpersonal relationships with their patients, capturing their learning needs, facilitating an effective dialogue and providing individualized patient-centered education (Svavarsdottir, Sigurdardottir, \& Steinsbekk, 2015).

This study also supports the conclusion from a recent meta-synthesis (Schulman-Green et al., 2016) in that knowledge about disease processes and the role of medications is essential to successful self-management. The informants in this study highlighted that knowledge about medications was crucial for their ability to self-manage right after they had attended the nurse-led PE intervention (Grønning, Midttun, \& Steinsbekk, 2016) because this knowledge made the participants more confident in taking their medications as prescribed. Knowledge about the disease process, particularly about the immune system and possible dangerous interactions between herbs and DMARDs was the knowledge that had made a significant impression. The informant that talked about this example was an experienced patient, diagnosed several years ago, and previously tried different DMARDs. This finding highlights the importance of providing patients with disease related information throughout the disease course as the EULAR recommendations for patient education for people with inflammatory arthritis state (Zangi et al., 2015). Patients should be offered PE at a minimum in the early stages of the disease, and when new pharmacological treatment is initiated.

This study verified that effective medications improve patients' quality of life (Scott, Ibrahim, Lewis, Scott, \& Strand, 2016; Strand et al., 2012) since the informants clearly spoke about that having a good life depended on effective medications. The RCT that nested this study (Gronning, Rannestad, Skomsvoll, Rygg, \& Steinsbekk, 2014) showed a significant reduction in the objective measure of disease activity, DAS28-3, from baseline to 12 months in the group of patients who attended the nurse-led PE intervention. The DAS28-3 score is monitoring treatment response (van Riel \& Renskers, 2016), and it is likely to assume that a decrease in disease activity is associated with effective medications. For medications to be effective, they are dependent on patients taking them as prescribed. The informants stated that they were mostly satisfied with their lives and that they had little symptoms. These statements may elaborate a bit about how the informants perceived the reduction in DAS28-3. Also, the informants had previously underlined that they had learned about the importance of taking medications as prescribed in the nurse-led PE intervention (Gronning et al., 2016).

The main strength of this study is that it is the first study that explores patients' experiences of participating in nurse-led PE one year after, and how participation may have influenced patients' lives, resources, and skills to manage the disease. However, this design also introduces a major limitation, the risk of recall bias. The patients may not have remembered what they actually learned and discussed in the nurse-led PE 
intervention. It was evident from the interviews that some informants could not pinpoint the exact influence of the intervention on their current situation. To reduce recall bias, data from previous interviews were used to remind the informants of what they had said earlier. In some parts of the interviews, it was not clear if the informants related their descriptions to the PE or not. There were also a few parts which were not clear if the interviewer asked directly about the influence of the PE.

Nevertheless, the results related to the influence of the PE are based on the instances where it was possible to distinguish this. Another strength is the transferability of the findings. A rich description of the context and the participants is provided (Malterud, 2001), and the knowledge is applicable to patients with other chronic diseases as well (Stenberg et al., 2016). The themes in this nurse-led PE intervention are similar to those delivered in PE interventions targeting patients with other chronic illnesses.

In qualitative studies, it is also a risk of personal bias or interpretations during the interview process. To reduce this risk, all authors participated in the analyses process and discussed the possible influence of the research team's background, position and already existing knowledge about the topics related to the analysis process (Malterud, 2001). There is also a risk of selection bias due to only persons agreeing to participate in the RCT were included. However, the sample was heterogeneous with persons having different experiences, gender, ages and variations in diagnosis, disease duration, and disease severity. The characteristics of the informants are similar to other populations of patients with arthritis (Gronning et al., 2010).

\section{CONCLUSION}

The informants in this study reported that participating in the nurse-led PE intervention helped them discover new opportunities and perspectives in creating and having a good life when living with arthritis. The most important experience from the PE intervention was exchanging experiences with fellow patients. Clinical nurses ought to be aware of what patients consider as having a good life to support patients to take more control over their own health and environments and to make choices conducive to their health.

\section{REFERENCES}

Albano, M. G., Giraudet-Le Quintrec, J. S., Crozet, C., \& d'Ivernois, J. F. (2010). Characteristics and development of therapeutic patient education in rheumatoid arthritis: analysis of the 2003-2008 literature. Joint Bone Spine, 77, 405-410.

Anderson, R. M., \& Funnell, M. M. (2010). Patient Empowerment: Myths and Misconceptions. Patient Educ Couns, 79, 277-282. doi:10.1016/j.pec.2009.07.025

Barlow, J., Turner, A., Swaby, L., Gilchrist, M., Wright, C., \& Doherty, M. (2009). An 8 -yr follow-up of arthritis self-management programme participants. Rheumatology (Oxford), 48, 128-133.

Bergsten, U., Bergman, S., Fridlund, B., \& Arvidsson, B. (2011). "Striving for a good life" - the management of rheumatoid arthritis as experienced by patients. Open Nursing Journal, 5, 95-101. 
Burton, W., Morrison, A., Maclean, R., \& Ruderman, E. (2005). Systematic review of studies of productivity loss due to rheumatoid arthritis. Occupational Medicine, 56, 18-27. doi:10.1093/occmed/kqi171

Cottrell, J. E. D., Jonas, M., Bergsten, U., Blaas, E., de la Torre Aboki, J., Howse, C., . . . Bulinckx, L. (2012). The Nurse's Role in Addressing Unmet Treatment and Management Needs of Patients With Rheumatoid Arthritis: Delphi-Based Recommendations. International Journal of Nursing Knowledge, n/a-n/a. doi:10.1111/j.2047-3095.2012.01231.x

Daker-White, G., Donovan, J., \& Campbell, R. (2014). Redefined by illness: metaethnography of qualitative studies on the experience of rheumatoid arthritis. Disabil Rehabil, 36, 1061-1071. doi:doi:10.3109/09638288.2013.829531

de Ridder, D., Geenen, R., Kuijer, R., \& van Middendorp, H. (2008). Psychological adjustment to chronic disease. The Lancet, 372, 246-255.

Dures, E., Almeida, C., Caesley, J., Peterson, A., Ambler, N., Morris, M., . . . Hewlett, S. (2016). Patient preferences for psychological support in inflammatory arthritis: a multicentre survey. Ann Rheum Dis, 75, 142-147. doi:10.1136/annrheumdis-2014-205636

Dures, E., Hewlett, S., Ambler, N., Jenkins, R., Clarke, J., \& Gooberman-Hill, R. (2016). A qualitative study of patients' perspectives on collaboration to support self-management in routine rheumatology consultations. BMC Musculoskelet Disord, 17, 129. doi:10.1186/s12891-016-0984-0

Dures, E., Kitchen, K., Almeida, C., Ambler, N., Cliss, A., Hammond, A., . . Hewlett, S. (2012). "They didn't tell us, they made us work it out ourselves": patient perspectives of a cognitive-behavioral program for rheumatoid arthritis fatigue. Arthritis Care Res (Hoboken), 64, 494-501. doi:10.1002/acr.21562

Grønning, K., Midttun, L., \& Steinsbekk, A. (2016). Patients' confidence in coping with arthritis after nurse-led education; a qualitative study. BMC Nurs, 15, 28. doi:10.1186/s12912-016-0150-x

Grønning, K., Rannestad, T., Skomsvoll, J. F., Rygg, L. O., \& Steinsbekk, A. (2014). Long-term effects of a nurse-led group and individual patient education programme for patients with chronic inflammatory polyarthritis - a randomised controlled trial. J Clin Nurs, 23(7-8), 1005-1017. doi:10.1111/jocn.12353

Grønning, K., Rødevand, E., \& Steinsbekk, A. (2010). Paid work is associated with improved health-related quality of life in patients with rheumatoid arthritis. Clin Rheumatol. doi: DOI 10.1007/s10067-010-1534-z

Grønning, K., Lomundal, B., Koksvik, H. S., \& Steinsbekk, A. (2011). Coping with arthritis is experienced as a dynamic balancing process. A qualitative study. Clin Rheumatol, 30, 1425-1432. doi:10.1007/s10067-011-1836-9

Grønning, K., Midttun, L., \& Steinsbekk, A. (2016). Patients' confidence in coping with arthritis after nurse-led education: a qualitative study. BMC Nursing, 15, 28. doi:10.1186/s12912-016-0150-x

Grønning, K., Rannestad, T., Skomsvoll, J. F., Rygg, L. O., \& Steinsbekk, A. (2013). Long-term effects of a nurse-led group and individual patient education programme for patients with chronic inflammatory polyarthritis: a randomised controlled trial. J Clin Nurs. doi:10.1111/jocn.12353 
Grønning, K., Skomsvoll, J. F., Rannestad, T., \& Steinsbekk, A. (2012). The effect of an educational programme consisting of group and individual arthritis education for patients with polyarthritis: A randomised controlled trial. Patient Educ Couns, 88, 113-120. doi:10.1016/j.pec.2011.12.011

Henderson, V. (2006). The concept of nursing. 1977. J Adv Nurs, 53(1), 21-31; discussion 32-24. doi:10.1111/j.1365-2648.2006.03660.x

Holland, P., \& Collins, A. M. (2016). "Whenever I can I push myself to go to work": a qualitative study of experiences of sickness presenteeism among workers with rheumatoid arthritis. Disabil Rehabil, 1-10. doi:10.1080/09638288.2016.1258436

Lempp, H., Scott, D., \& Kingsley, G. (2006). The personal impact of rheumatoid arthritis on patients' identity: a qualitative study. Chronic Illn, 2, 109-120.

Lindstrom, B., \& Eriksson, M. (2005). Salutogenesis. J Epidemiol Community Health, $59,440-442$.

Malterud, K. (2001). Qualitative research: standards, challenges, and guidelines. Lancet, $358,483-488$.

Malterud, K. (2012). Systematic text condensation: a strategy for qualitative analysis. Scand J Public Health, 40, 795-805. doi:10.1177/1403494812465030

Poh, L. W., He, H. G., Chan, W. C., Lee, C. S., Lahiri, M., Mak, A., \& Cheung, P. P. (2016). Experiences of Patients With Rheumatoid Arthritis: A Qualitative Study. Clin Nurs Res. doi:10.1177/1054773816629897

QSR, I. (2007). NVIVO (Version 9). Victoria: QSR International.

Ring Jacobsson, L., Milberg, A., Hjelm, K., \& Friedrichsen, M. (2016). Gaining perspective on own illness - the lived experiences of a patient education programme for women with treated coeliac disease. $J$ Clin Nurs. doi:10.1111/jocn.13123

Schulman-Green, D., Jaser, S. S., Park, C., \& Whittemore, R. (2016). A meta-synthesis of factors affecting self-management of chronic illness. $J$ Adv Nurs. doi:10.1111/jan.12902

Scott, I. C., Ibrahim, F., Lewis, C. M., Scott, D. L., \& Strand, V. (2016). Impact of intensive treatment and remission on health-related quality of life in early and established rheumatoid arthritis. RMD Open, 2, e000270.

Sharpe, L. (2016). Psychosocial management of chronic pain in patients with rheumatoid arthritis: challenges and solutions. J Pain Res, 9, 137-146. doi:10.2147/jpr.s83653

Stenberg, U., Haaland-Overby, M., Fredriksen, K., Westermann, K. F., \& Kvisvik, T. (2016). A scoping review of the literature on benefits and challenges of participating in patient education programs aimed at promoting self-management for people living with chronic illness. Patient Educ Couns. doi:10.1016/j.pec.2016.07.027

Strand, V., Rentz, A. M., Cifaldi, M. A., Chen, N., Roy, S., \& Revicki, D. (2012). Health-related quality of life outcomes of adalimumab for patients with early rheumatoid arthritis: results from a randomized multicenter study. J Rheumatol, 39, 63-72. doi:10.3899/jrheum.101161

Svavarsdottir, M. H., Sigurdardottir, A. K., \& Steinsbekk, A. (2015). What is a good educator? A qualitative study on the perspective of individuals with coronary heart disease. Eur J Cardiovasc Nurs. doi:10.1177/1474515115618569 
van Eijk-Hustings, Y., van Tubergen, A., Bostrom, C., Braychenko, E., Buss, B., Felix, J., . . . Hill, J. (2011). EULAR recommendations for the role of the nurse in the management of chronic inflammatory arthritis. Ann Rheum Dis. doi:10.1136/annrheumdis-2011-200185

van Riel, P. L., \& Renskers, L. (2016). The Disease Activity Score (DAS) and the Disease Activity Score using 28 joint counts (DAS28) in the management of rheumatoid arthritis. Clin Exp Rheumatol, 34, 40-S44.

WHO. (1986). Ottawa Charter for Health Promotion. Paper presented at the International Conference on Health Promotion, Ontario Canada.

Zangi, H. A., Ndosi, M., Adams, J., Andersen, L., Bode, C., Bostrom, C., . . . van Tubergen, A. (2015). EULAR recommendations for patient education for people with inflammatory arthritis. Ann Rheum Dis. doi:10.1136/annrheumdis-2014206807

Zuidema, R. M., Repping-Wuts, H., Evers, A. W., Van Gaal, B. G., \& Van Achterberg, T. (2015). What do we know about rheumatoid arthritis patients' support needs for self-management? A scoping review. Int J Nurs Stud, 52(10), 1617-1624. doi:10.1016/j.ijnurstu.2015.05.008 